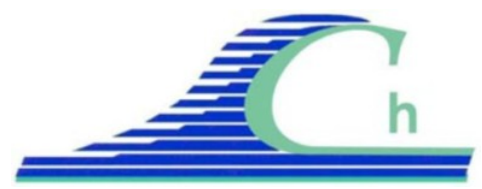

XII ${ }^{\text {ìmes }}$ Journées Nationales Génie Côtier - Génie Civil

Cherbourg, 12-14 juin 2012

DOI:10.5150/jngcgc.2012.090-M @ Editions Paralia CFL

disponible en ligne - http://www.paralia.fr - available online

\title{
Étude numérique du comportement d'hydroliennes à axe horizontal : vers une modélisation d'un parc d'hydroliennes
}

\author{
Paul MYCEK ${ }^{1,2}$, Benoît GAURIER ${ }^{2}$, Grégory GERMAIN ${ }^{2}$, \\ Grégory PINON ${ }^{1}$, Élie RIVOALEN ${ }^{1,3}$
}

1. Université du Havre, UFR Sciences et Techniques, LOMC, UMR 6294, CNRS Université du Havre, 53 rue de Prony, BP 540, 76058 Le Havre, France. gregory.pinon@univ-lehavre.fr

2. IFREMER, Centre Manche Mer du Nord, Service Hydrodynamique et Océanométéo, 150 quai Gambetta, BP 699, 62321 Boulogne-sur-Mer, France.

\{paul.mycek, benoit.gaurier,gregory.germain\}@ifremer.fr

3. INSA de Rouen, EA 3828 - LOFIMS,

Avenue de l’Université, BP 08, 76801 Saint-Étienne-du-Rouvray, France.

elie.rivoalen@insa-rouen.fr

\section{Résumé :}

L'implantation et le développement de fermes d'hydroliennes sont soumis à la compréhension des effets d'interaction entre machines. Des essais expérimentaux ont été réalisés dans le canal à houle et courant de l'IFREMER à Boulogne-sur-Mer, sur des maquettes d'hydrolienne 3 pales à axe horizontal, dans le but de caractériser les interactions entre deux hydroliennes alignées avec le courant.

En parallèle un code numérique tridimensionnel est développé pour modéliser l'écoulement derrière une hydrolienne. Ce code de calcul est basé sur la méthode Vortex. Il permet entre autres de calculer les efforts et moments sur le rotor ainsi que de tracer des cartes de vitesses en aval de l'hydrolienne.

La plupart des études numériques portant sur les interactions entre hydroliennes ne considèrent en réalité qu'une seule hydrolienne, les conditions d'entrée étant issues de l'écoulement calculé à partir d'une autre simulation indépendante. Par ailleurs, les modèles numériques prenant effectivement en compte les effets d'interaction s'appuient en général sur l'approximation du disque poreux. Le code de calcul développé dans le cadre de cette étude permet de considérer une ferme d'hydroliennes dans son ensemble. Nous présentons les premiers résultats numériques et validations obtenus dans ce cadre.

\section{Mots-clés :}

Énergies marines - Hydroliennes - Simulation numérique - Méthode vortex - Méthode lagrangienne - Essais expérimentaux

\section{Introduction}

L'implantation de la première hydrolienne en mer, la turbine Seagen de MCT (Marine Current Turbine), installée depuis 2008 dans le Strangford narrows en Irlande du Nord, 


\section{Thème 5 - Énergies marines}

a été un élément fondateur pour cette technologie. Depuis, plusieurs hydroliennes ont été installées et les premiers développements de parcs sont aujourd’hui envisagés. En France notamment, quatre turbines Open Hydro ont été installées au second semestre 2011 près de Paimpol. Ces développements nécessitent une caractérisation et une évaluation précise des effets d'interaction entre machines pour optimiser au mieux la gestion de l'espace.

Des essais expérimentaux d'interactions entre hydroliennes ont été menés, notamment grâce à des disques poreux (HARRISON et al., 2010) ou grâce à des maquettes de turbines réelles (MYCEK et al., 2011). Dans ces deux dernières études, les turbines sont alignées et il n’y a pas pour l'instant d'études expérimentales avec des implantations spatiales réellement 2D. Compte tenu de la taille des installations expérimentales et de la complexité des configurations possibles d'implantation des turbines (MYERS et al., 2010), il ne sera pas possible d'évaluer ces effets d'interaction dans des infrastructures expérimentales classiques.

Le recours à des outils numériques dédiés est de ce fait indispensable. Outre la détermination précise des coefficients de puissance et de traînée d'une hydrolienne (BALTAZAR \& FALCÃO DE CAMPOS, 2008), ces outils doivent permettre la simulation du sillage de ce type de turbine. Ainsi, à terme, il sera possible de faire interagir plusieurs hydroliennes entre elles. A l'heure actuelle, les logiciels de type BEM (Boundary Element Momentum theory) ne permettent pas de simuler proprement les sillages (BALTAZAR \& FALCÃO DE CAMPOS, 2008). Nous proposons ici de présenter les développements numériques entrepris pour simuler le comportement global, en terme de rendement et de sillage, d'une hydrolienne.

Dans une première partie, nous présentons la méthode numérique utilisée. Dans une seconde partie, nous présentons les résultats de validation obtenus sur deux géométries d'hydroliennes (BAHAJ et al., 2007, MYCEK et al., 2011). Enfin, nous présenterons les développements numériques entrepris pour traiter les interactions entre plusieurs machines positionnées dans un espace proche.

\section{Méthode numérique utilisée}

Le code de simulation numérique de l'écoulement autour d'une hydrolienne est basé sur une méthode Lagrangienne particulaire et présenté en détail dans (PINON et al., 2005 ; PINON et al., 2012). Les équations de Navier-Stokes sont formulées en vitesse / vorticité $(\boldsymbol{u}, \boldsymbol{\omega})$ pour un fluide Newtonien incompressible :

$\nabla \cdot \boldsymbol{u}=0$

$\frac{D \omega}{D t}=(\omega \cdot \nabla) \boldsymbol{u}+v \Delta \boldsymbol{\omega}$,

où $\boldsymbol{u}$ est la vitesse du fluide, $\boldsymbol{\omega}=\nabla \wedge \boldsymbol{u}$ est la vorticité et $v$ la viscosité cinématique.

$\boldsymbol{u}=\nabla \wedge \boldsymbol{\psi}+\nabla \phi+\boldsymbol{u}^{\infty}=\boldsymbol{u}^{\psi}+\boldsymbol{u}^{\phi}+\boldsymbol{u}^{\infty}$, 


\section{XII ${ }^{\text {èmes }}$ Journées Nationales Génie Côtier - Génie Civil \\ Cherbourg, 12-14 juin 2012}

La décomposition de Helmholtz de la vitesse (eq. (3)) permet de scinder la composante de vitesse en une composante potentielle $\boldsymbol{u}^{\phi}$ (elle dérive du potentiel scalaire $\phi$ ), une composante tourbillonnaire ou rotationelle $\boldsymbol{u}^{\psi}$ obtenue à partir du potentiel vecteur $\boldsymbol{\psi}$ et une partie constante $\boldsymbol{u}^{\infty}$. Physiquement, $\boldsymbol{u}^{\phi}$ représente la perturbation de vitesse liée à la présence du rotor de l'hydrolienne, $\boldsymbol{u}^{\prime \prime}$ représente la présence du sillage tourbillonnaire qui se développe derrière l'hydrolienne et $\boldsymbol{u}^{\infty}$ représente le courant incident, supposé constant dans cette étude. Par combinaison des équations (1) et (3), nous obtenons les relations suivantes :

$\Delta \psi=-\omega$

$\Delta \phi=0$.

Les équations (4) et (5), nous permettent d'obtenir les formes de $\boldsymbol{u}^{\phi}$ et $\boldsymbol{u}^{\psi}$. La figure 1(a) représente schématiquement les différents domaines de la méthode utilisée.

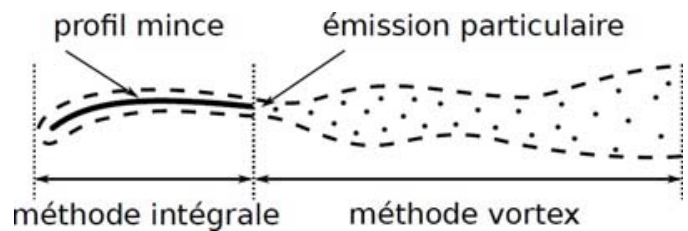

(a) Description schématique des méthodes mises en jeu

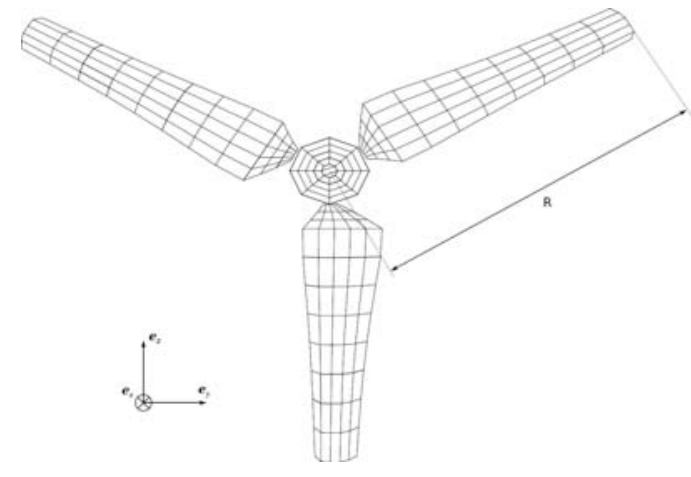

(b) Exemple de maillage d'une hydrolienne

Figure 1. Description de la méthode numérique.

\subsection{Composante potentielle de la vitesse}

L'hydrolienne (les 3 pales et la partie centrale) est prise en compte par une méthode intégrale de frontière (cf. partie gauche de la figure 1(a)) nous permettant d'obtenir la composante $\boldsymbol{u}^{\phi}$. Cette hydrolienne est discrétisée en $N_{p}$ éléments surfaciques comme le présente le schéma de la figure 1(b). Une répartition de doublets surfaciques $\mu$ impose une vitesse normale nulle sur les parois. Grâce à l'équation (5) et pour $M$ un point quelconque de l'espace, nous obtenons alors la perturbation de vitesse liée à la présence du rotor par :

$\boldsymbol{u}^{\phi}(M)=\frac{1}{4 \pi} \sum_{p=1}^{N_{p}} \mu_{p} \nabla_{M}\left(\frac{\boldsymbol{M P} \cdot \boldsymbol{n}(P)}{|\boldsymbol{M P}|^{3}}\right) d s_{p}$

où $P$ est le centre d'une facette quelconque de l'hydrolienne, facette de normale $\boldsymbol{n}(P)$, de surface $\phi_{p}$ et de doublet normal $\mu_{p}$ supposé constant sur la facette. 


\subsection{Composante tourbillonnaire de la vitesse}

A partir de l'équation (4) et en utilisant la relation de Biot \& Savart (eq. (7)), nous obtenons la composante rotationelle de la vitesse $\boldsymbol{u}^{\psi}(M)$ :

$\boldsymbol{u}^{\psi}(M)=\frac{1}{4 \pi} \int_{v} \boldsymbol{K}_{\varepsilon}\left(\boldsymbol{M} \boldsymbol{M}^{\prime}\right) \wedge \boldsymbol{\omega}\left(\boldsymbol{M}^{\prime}\right) d v^{\prime}$,

où $M^{\prime}$ est un autre point quelconque de l'espace et $\boldsymbol{K}_{\varepsilon}$ (eq. (8)) représente le noyau régularisé de Biot \& Savart.

$$
\boldsymbol{K}_{\varepsilon}\left(\boldsymbol{M} \boldsymbol{M}^{\prime}\right)=\frac{M \boldsymbol{M}^{\prime}}{\left(\left|\boldsymbol{M} \boldsymbol{M}^{\prime}\right|^{2}+\varepsilon^{2}\right)^{3 / 2}} .
$$

L’intégrale précédente (eq.(7)) est par la suite discrétisée en une somme de contributions des particules représentant l'écoulement rotationnel (cf. partie droite de la figure 1(a)). Les particules sont crées à chaque pas de temps au bord de fuite des pales grâce à la condition de Kutta-Joukovsky. Ces particules sont advectées grâce à leur vitesse évaluée à partir de l'équation (3), l'intégration en temps se faisant par un schéma Runge-Kutta d'ordre 2. Une grande partie des détails de la méthode est disponible dans (PINON et al., 2012).

\section{Résultats numériques}

Le comportement d'une hydrolienne est influencé par différents paramètres, parmi lesquels :

- La norme de la vitesse amont, uniforme dans la présente étude, notée $U_{\infty}=\left|\boldsymbol{u}^{\infty}\right|$;

- Le Tip Speed Ratio (TSR), défini comme le ratio entre la vitesse en bout de pale et la vitesse de l'écoulement amont $U_{\infty}$ :

$$
T S R=\frac{\Omega R}{U_{\infty}}
$$

où $R=D / 2$ est le rayon du rotor et $\Omega$ est sa vitesse de rotation.

Tableau 1. Caractéristiques de la maquette d'hydrolienne.

\begin{tabular}{lll}
\hline Caractéristique & IFREMER LOMC & BBMC \\
\hline Profil des pales & NACA 63418 & NACA 63-8XX \\
Diamètre du rotor $(D)$ & $700 \mathrm{~mm}$ & $800 \mathrm{~mm}$ \\
Diamètre de la nacelle & $92 \mathrm{~mm}$ & $100 \mathrm{~mm}$ \\
Longueur de la nacelle & $720 \mathrm{~mm}$ & - \\
Angle de calage (Pitch) & $0^{\circ}$ & $0^{\circ}, 5^{\circ} 10^{\circ}$ et $13^{\circ}$ \\
TSR étudiés & {$[0 ; 10]$} & {$[2 ; 10]$} \\
Sens de rotation & Antihoraire & Horaire \\
Reynolds $\left(\operatorname{Re}_{\infty}=U_{\infty} R / v\right)$ & $\approx 280000$ & $\approx 580000$ \\
\hline
\end{tabular}




\section{XII ${ }^{\text {èmes }}$ Journées Nationales Génie Côtier - Génie Civil \\ Cherbourg, 12-14 juin 2012}

Des essais ont été réalisés dans le bassin d'essais de l'IFREMER de Boulogne-sur-Mer sur une maquette à l'échelle 1/30 d'un prototype d'hydrolienne, notée par la suite hydrolienne IFREMER-LOMC (MAGANGA et al., 2010 ; MYCEK et al., 2011). La description générale de la maquette est donnée par le tableau 1. Dans le cadre d'une validation plus contraignante, nous avons voulu confronter notre code à une autre configuration d'hydrolienne, dénommée par la suite hydrolienne BBMC et dont les résultats sont disponibles dans la littérature (BAHAJ et al., 2007).

\subsection{Les performances}

Les performances d'une hydrolienne peuvent être évaluées classiquement par le calcul du coefficient de puissance $C_{P}$ et de traînée $C_{T}$ définis par :

$$
\begin{aligned}
C_{P} & =\frac{M_{x} \Omega}{\frac{1}{2} \rho \pi R^{2} U_{\infty}^{3}} . \\
C_{T} & =\frac{F_{X}}{\frac{1}{2} \rho \pi R^{2} U_{\infty}^{2}}
\end{aligned}
$$

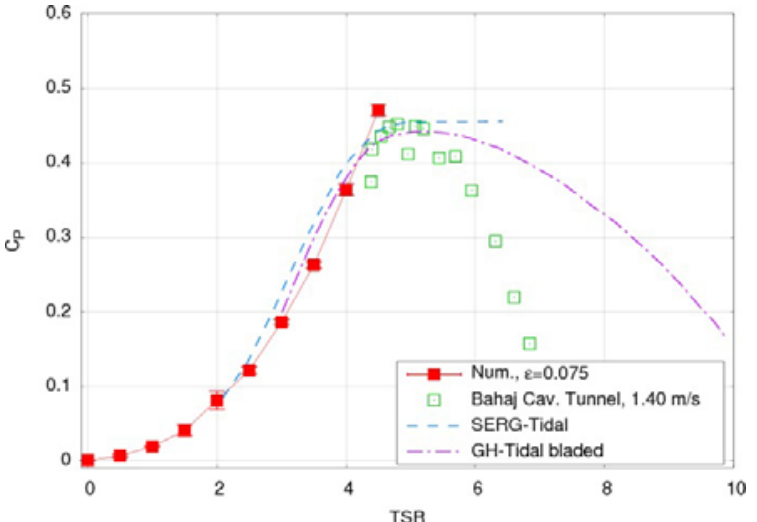

(a) Angle de calage $0^{\circ}$

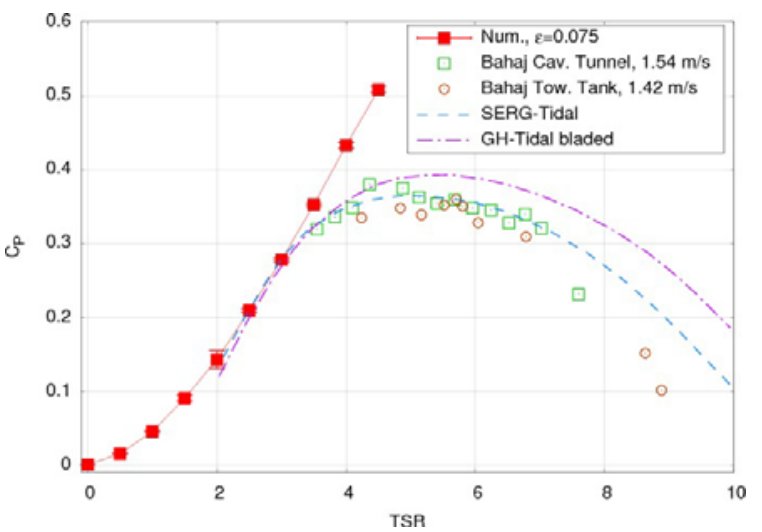

(c) Angle de calage $10^{\circ}$

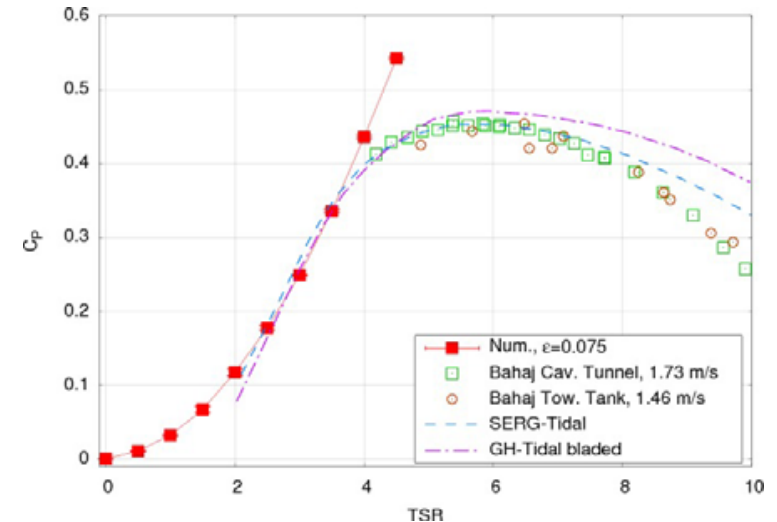

(b) Angle de calage $5^{\circ}$

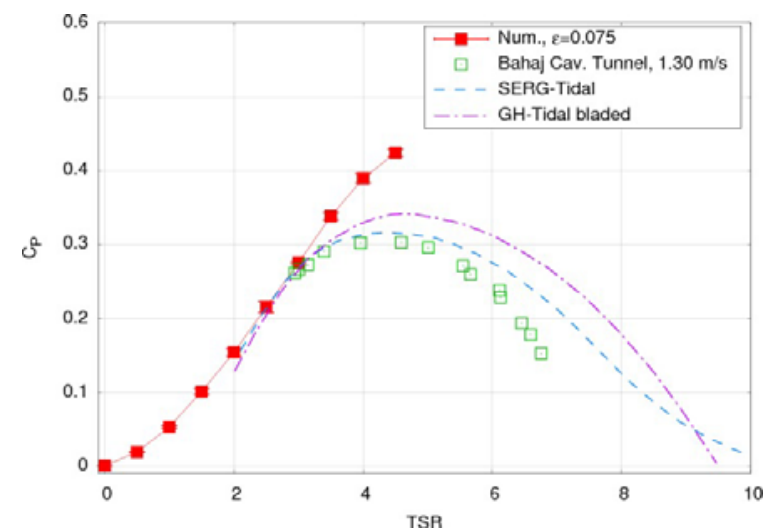

(d) Angle de calage $13^{\circ}$

Figure 2. Évolution des $C_{P}$ en fonction du TSR pour l'hydrolienne de type BBMC pour différents angles de calage (Pitch, voir tableau 1). 
La figure 2 montre l'évolution du coefficient de puissance de l'hydrolienne de type BBMC en fonction de son TSR pour différents angles de calage (cf. tableau 1). Les résultats numériques (SERG-Tidal et GH-Tidal Bladed) ainsi que les résultats expérimentaux ont été repris de (BAHAJ et al., 2007). Les résultats obtenus, pour un paramètre de discrétisation $\varepsilon=0.075$, sont en accord avec les résultats de la littérature, uniquement pour la partie ascendante de la courbe de $C_{P}$. Comme prévu, et lié au fait que nous émettons nos particules uniquement au bord de fuite par un modèle de type Kutta-Joukovsky, nous ne pouvons pas modéliser le décollement de couche limite. Ceci ne nous permet pas, pour le moment, de simuler correctement le comportement de l’hydrolienne pour des TSR élevés.

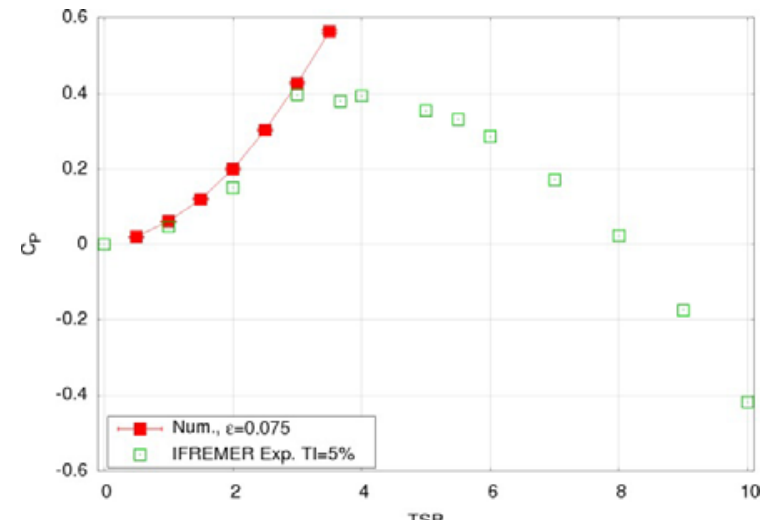

(a) $C_{P}$

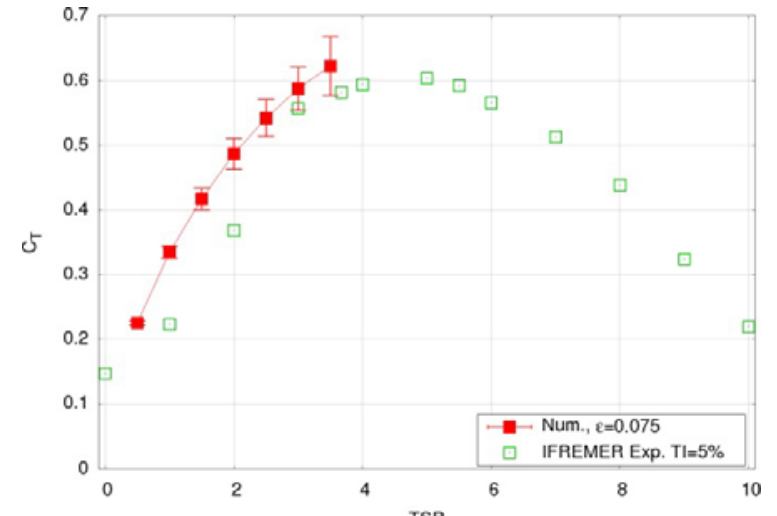

(b) ${ }_{T}^{\mathrm{TS}}$

Figure 3. Évolution du $C_{P}$ et du $C_{T}$ en fonction du TSR pour l'hydrolienne de type IFREMER-LOMC.

Les résultats numériques obtenus en $C_{P}$ et $C_{T}$ (le coefficient de traînée, eq.(11)) sur la configuration IFREMER-LOMC (figure 3) sont également en bon accord avec la même limitation lorsque les TSR augmentent. Nous pouvons en conclure que notre code prend en compte de manière satisfaisante différentes géométries pour la partie ascendante des courbes de coefficient de performance et traînée.

\subsection{Le sillage}

La figure 4(a) montre une carte de vitesse axiale moyenne en aval de l'hydrolienne IFREMER-LOMC obtenue numériquement. Elle est très similaire au résultats expérimentaux obtenus à l'IFREMER de Boulogne-sur-Mer grâce à un système de LDV (Laser Doppler Velocimetry), (MAGANGA et al., 2010 ; MYCEK et al., 2011). Expérimentalement, le taux de turbulence ambiant (TI) de l'écoulement incident est de 5\% (figure 4(a) en haut), alors que numériquement (figure 4(a) en bas), la présence d’un taux de turbulence ambiant ne peut pas être prise en compte dans le modèle actuellement. 


\section{XII ${ }^{\text {èmes }}$ Journées Nationales Génie Côtier - Génie Civil \\ Cherbourg, 12-14 juin 2012}

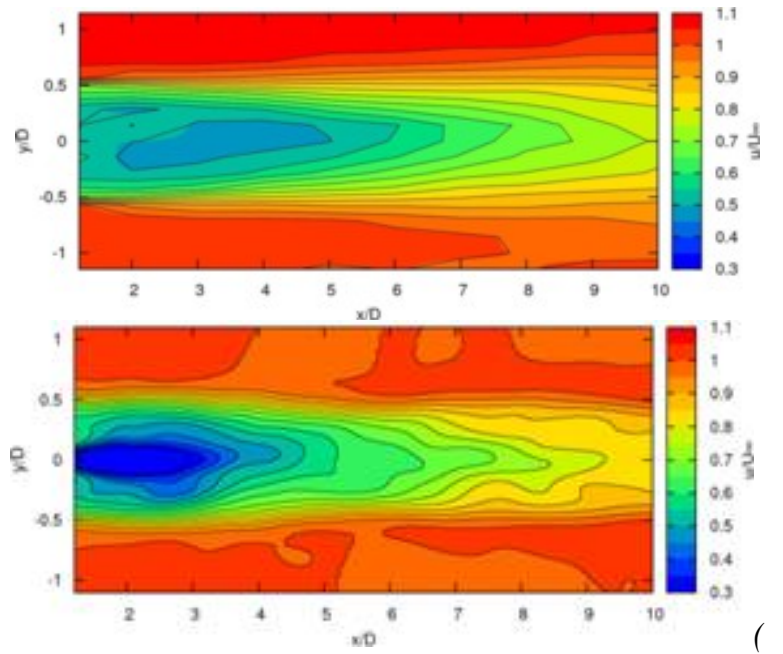

a) Carte de vitesse axiale obtenue expérimentalement (en haut) et numériquement (en bas)

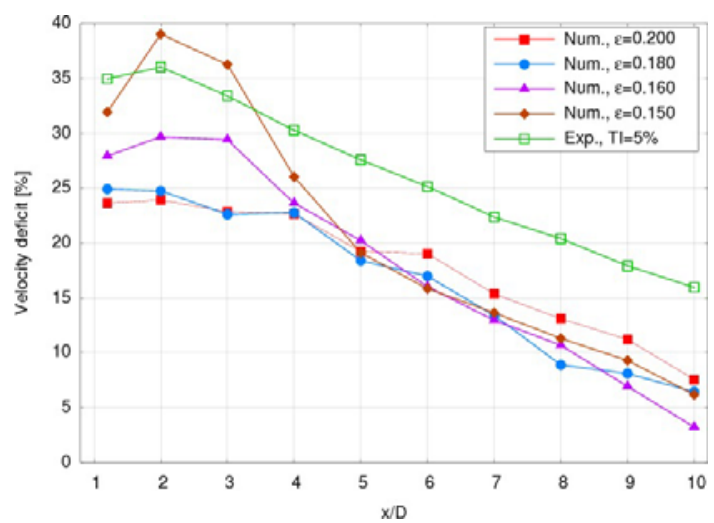

(b) Évolution du déficit de vitesse axiale

Figure 4. Caractérisation numérique du sillage à TSR=3,67.

À partir de ces cartes de vitesse, nous pouvons estimer la valeur moyenne de la vitesse axiale $\hat{u}$ à une certaine distance $x$ derrière l'hydrolienne en intégrant la vitesse axiale à cette distance sur l'aire d'influence de rayon $R^{*}$ de l'hydrolienne :

$\hat{u}(x)=\frac{1}{R^{*}} \int_{-R^{*}}^{+R^{*}}|y| u(x, y) d y$

Ici, $R^{*}$ est pris légèrement plus grand que $R$ de manière à ce que tout le déficit de vitesse induit par la présence de l'hydrolienne soit bien pris en compte. Le déficit moyen en $x$ est alors naturellement défini par

$\gamma(x)=100 \times\left(1-\hat{u}(x) / U_{\infty}\right)$

Cette quantité nous permet de mieux comparer nos résultats numériques et expérimentaux. La figure 4 montre une carte de vitesse axiale obtenue numériquement pour une configuration correspondant à un TI de $0 \%$ (figure 4(a)) et la comparaison du déficit de vitesse numérique derrière l’hydrolienne avec les résultats expérimentaux (figure 4(b)) et ce en fonction de différents paramètres de discrétisation $\varepsilon$. Les résultats numériques sont en bon accord avec l'expérience. Les différences observées proviennent pour partie du fait que le taux de turbulence ambiant (TI) ne peut pas encore être pris en compte dans le code numérique ainsi que du fait que le modèle de turbulence implémenté dans le code peut encore être amélioré. En effet, nous utilisons la méthode PSE pour prendre en compte la viscosité dans notre code et nous avons ajouté un modèle de turbulence de type LES (Large Eddy Simulation). Ce dernier est assez rudimentaire et il semble être très dissipatif, ce qui pourrait être un élément d'explication de nos différences avec l'expérimental. 


\subsection{Interactions entre hydroliennes}

La figure 5 présente les premiers résultats d'une simulation de 8 hydroliennes en interaction. Cette simulation est purement démonstrative et a pour objectif de démontrer la faisabilité de notre étude visant à modéliser les effets d'interaction de plusieurs hydroliennes en un espace proche. Dans la simulation présentée, la discrétisation des pales n'est pas assez fine pour pouvoir garantir la convergence des résultats en terme de coefficient de performance et de traînée. De même, pour des raisons de temps CPU trop important, la simulation n'a pas été menée assez longtemps pour avoir une bonne évaluation du sillage. Des travaux sont en cours sur notre code pour optimiser la demande mémoire et accélérer les calculs, par une optimisation scalaire et une meilleure parallélisation.

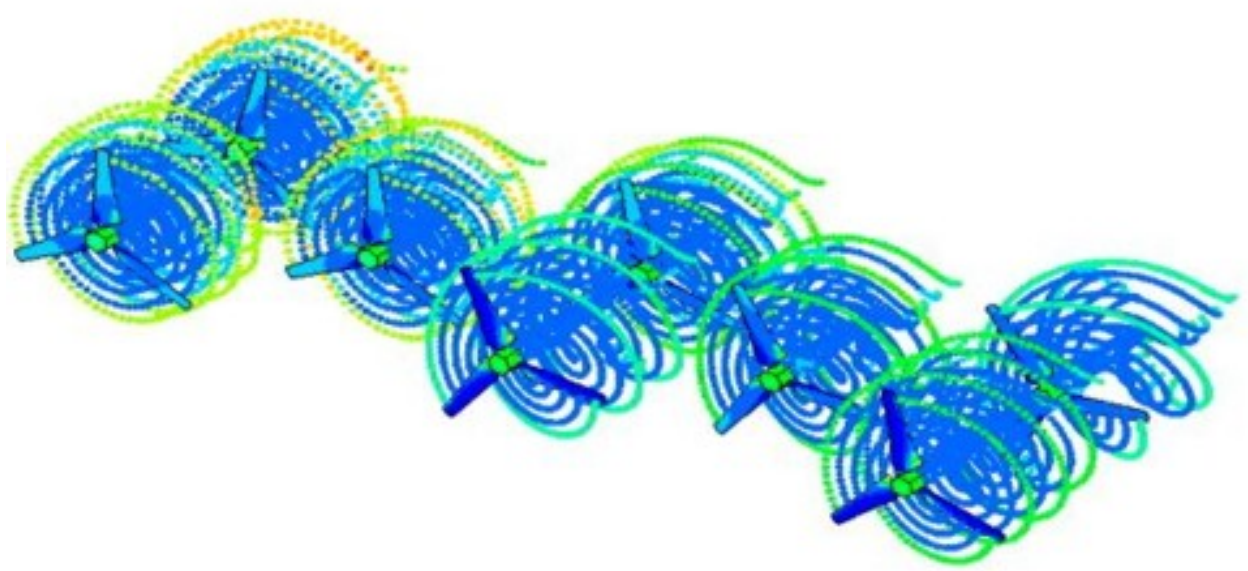

Figure 5. Simulation de 8 hydroliennes en interaction.

\section{Conclusion et perspectives}

Cette étude présente de manière succincte la méthode numérique utilisée pour obtenir dans une même simulation les coefficients de performance $C_{P}$ et de trainée $C_{T}$ d'une hydrolienne ainsi que la simulation du sillage de celle-ci. Nous voyons que les résultats sont en accord avec la littérature et les résultats expérimentaux réalisés par la même équipe. En ce qui concerne les performances des machines $\left(C_{P}\right.$ et $\left.C_{T}\right)$, les résultats obtenus sont bons et ce quelle que soit la géométrie et les angles de calage des pales mais uniquement pour la partie ascendante de la courbe de performance, c'est-à-dire pour des TSR modérés. Une amélioration est prévue pour pouvoir modéliser l'ensemble de la courbe et ainsi élargir la gamme de TSR.

En ce qui concerne les sillages, les résultats sont très prometteurs et des comparaisons avec les résultats expérimentaux sont très encourageants. Néanmoins, des améliorations en terme de prise en compte de la viscosité et du modèle de turbulence sont prévues pour améliorer les résultats. 


\section{XII ${ }^{\text {èmes }}$ Journées Nationales Génie Côtier - Génie Civil \\ Cherbourg, 12-14 juin 2012}

Les prochains travaux vont se concentrer sur des optimisations en terme de mémoire et accélération des calculs, par une optimisation scalaire et une meilleure parallélisation. Le but étant, in fine, de modéliser de manière réaliste l'interaction de plusieurs hydroliennes dans un espace proche, similaire aux fermes d'hydroliennes qui sont actuellement en prévision sur nos côtes.

\section{Remerciements}

Les auteurs souhaitent remercier la Région Haute-Normandie dans le cadre du programme RHYNO, ainsi que pour le soutien financier accordé au co-financement de la thèse de P. Mycek. Nous souhaitons aussi remercier le CRIHAN pour la mise à disposition des moyens informatiques pour les calculs numériques. Le code de simulation numérique a été initialement développé pour des applications aéronautiques dans le cadre de travaux avec l'entreprise Aircelle et la Région Haute-Normandie. Nous tenons également à remercier Thomas Bacchetti et Jean-Valéry Facq pour leur aide dans le présent projet.

\section{Références bibliographiques}

BAHAJ A, BATTEN W, MC CANN G.(2007) Experimental verifications of numerical predictions for the hydrodynamic performance of horizontal axis marine current turbines. Renewable Energy. doi:10.1016/j.renene. 2007.10.001

BALTAZAR J., FALCÃO DE CAMPOS J.A.C. (2008). Hydrodynamic analysis of a horizontal axis marine current turbine with a boundary element method. 27th OMAE Conference, Estoril (Portugal), pp 883-893.

BATTEN W., BAHAJ A., MOLLAND A., CHAPLIN J. (2008). The prediction of the hydrodynamic performance of marine current turbines. Renewable Energy. doi:10.1016/j.renene.2007.05.043

HARRISON M., BATTEN W., MYERS L., BAHAJ A. (2010). Comparison between CFD simulations and experiments for predicting the far wake of horizontal axis tidal turbines. IET Renew. Power Gener. Vol. 4 (6), pp 613-627. doi:10.1049/ietrpg.2009.0193

MAGANGA F., GERMAIN G., KING J., PINON G., RIVOALEN E. (2010). Experimental characterisation of flow effects on marine current turbine behaviour and on its wake properties. IET Renewable Power Generation, Vol. 4, n 6, pp 498-509. doi:10.1049/iet-rpg. 2009.0205

MYCEK P., GAURIER B., GERMAIN G., PINON G., RIVOALEN E. (2011). Numerical and experimental study of the interaction between two marine current turbines, Proceedings of the 9th European Wave and Tidal Energy Conference (EWTEC), Southampton, UK.

MYERS L., BAHAJ A., RETZLER C., RICCI P., DHEDIN J.-F. (2010). Inter-device spacing issues within wave and tidal energy converter arrays. $3^{\text {rd }}$ ICOE, Bilbao. 
Thème 5 - Énergies marines

PINON G., BRATEC H., HUBERSON S., PIGNOT G., RIVOALEN E. (2005). Vortex method for simulation of a $3 D$ round jet in a cross-stream. Journal of Turbulence, Vol. 6, n 18, pp 1-25.

PINON G., MYCEK P., GERMAIN G., RIVOALEN R (2012). Numerical Simulation of the Wake of Marine Current Turbines with a Particle Method. Renewable Energy doi:10.1016/j.renene.2012.03.037 